ISSN 1997-5902

\title{
Étude floristique et ethnobotanique de la flore médicinale dans la région du Gharb, Maroc
}

\author{
Lahcen BOUAYYADI, Mohamed EL HAFIAN, Lahcen ZIDANE \\ Laboratoire de Biodiversité et Ressources Naturelles, Université Ibn Tofaïl, Faculté des Sciences, BP 133, Kénitra, \\ Maroc. \\ Auteur de la correspondance : $\underline{\text { bouayyadilahcen76@gmail.com }}$
}

Original submitted in on $16^{\text {th }}$ June 2015. Published online at www.m.elewa.org on 30 th September 2015 http://dx.doi.org/10.4314/jab.v93i1.10

\section{RÉSUMÉ :}

Objectif : Une étude ethnobotanique des plantes médicinales a été menée dans la région du Gharb, Maroc dans le but de créer une base de données des espèces médicinales utilisées dans la pharmacopée traditionnelle par la population locale.

Méthodologie et résultats : A l'aide de 520 fiches questionnaires une série d'enquêtes ethnobotaniques a été réalisée sur le terrain pendant deux campagnes 2012/2013 - 2013/2014 auprès des usagers, des herboristes et tradipraticiens. Le dépouillement des données recueillies a permis d'inventorier 137 espèces appartenant à 116 genres et 57 familles dont les plus importantes par leur effectif spécifique sont : les Lamiaceae, les Apiaceae, les Asteraceae, les Fabaceae et les Poaceae. Les résultats de cette étude ont montré aussi que le feuillage constitue la partie la plus utilisée, et la majorité des remèdes est préparée sous forme de décoction et poudre et administrés exclusivement par voie orale. En plus les affections digestives, métaboliques et dermatologiques sont les plus fréquentes.

Conclusion et application de la recherche : Par ailleurs, ces résultats peuvent être considérés comme une source d'information pour les recherches scientifiques dans le domaine de la phytochimie, et de la pharmacologie.

Mots-clés : Ethnobotanique, Gharb, Plantes Médicinales, Médecine Traditionnelles.

\begin{abstract}
Objective: An ethnobotanical inventory was conducted in the Gharb region, Morocco in order to create a database of species of medicinal plants used in traditional medicine by the local populations.

Methods and results: By using 520 questionnaires, a series of ethnobotanical field surveys was conducted during two campaigns (2012/2013-2013/2014) with users of plants, herbalists and traditional healers. The counting of the collected results allowed the inventory of 137 species belonging to 116 genera and 57 families, among which the most important of the species are: Lamiaceae, Apiaceae, Asteraceae, Fabaceae and Poaceae. The results of this study have also shown that the foliage is the most used part, and that the majority of remedies are prepared as a decoction or powder, and exclusively administered by the oral route. Besides, the digestive, metabolic and dermatological affections are the most common.

Conclusion and application of research: Also, these results can be considered as a source of information for scientific research in the field of phytochemistry and pharmacology.
\end{abstract}

Key words : Ethnobotany, Gharb, Medicinal Plants, Traditional Medicine 


\section{INTRODUCTION}

Dès son apparition, il y a 3 millions d'années seulement, l'homme a utilisé les plantes à d'autres fins que de la nourriture. Que la plante soit comestible ou toxique, qu'elle serve à tuer le gibier et l'ennemi ou à soigner, l'homme a découvert par une suite d'échecs et de réussites, l'utilisation des plantes pour son mieux-être. Ainsi, même actuellement, malgré le progrès de la pharmacologie, l'usage thérapeutique des plantes médicinales est très présent dans certains pays du monde et surtout les pays en voie de développement, en l'absence d'un système médical moderne (Tabuti et al., 2003). La Méditerranée est un haut lieu incontesté de la biodiversité mondiale, en raison de la diversité et de la richesse de sa flore. En effet, le Bassin méditerranéen abrite environ 10 $\%$ (25000) des plantes vasculaires connues dans le monde (E.A. Radford et al., 2011) Le Maroc par sa position biogéographique, offre une très grande diversité écologique et floristique. II est l'un des pays méditerranéens qui ont une longue tradition médicale et un savoir-faire traditionnel à base de plantes médicinales (SCHERRER \& al., 2005). Ainsi, la flore sauvage du Maroc recèle 41 écosystèmes formés de plus de 4200 espèces sauvages dont 600 espèces ont des vertus aromatiques ou médicinales et seulement 80 espèces sont actuellement exploitées. Le développement des produits de terroir, notamment les plantes aromatiques et médicinales (PAM) est considérées comme un objectif privilégié dans la stratégie agricole marocaine. Le rôle socioéconomique des PAM n'est guère à démonter ; les recettes à l'exportation génèrent plus de 615 millions $\mathrm{DH}$ et offrent plus de 500.000 Journées de Travail avec un revenu global de 25 millions DH (APDN., 2012). La contribution

\section{PRESENTATION DE LA ZONE D'ETUDE}

La région du Gharb-Chrarda-Beni Hssen se situe dans la partie Nord-Ouest du Royaume. Elle est limitée à l'Ouest par l'Océan Atlantique, au Nord par la région de TangerTétouan, à l'Est par les deux régions de Taza-Al Hoceima-Taounate et de Fès-Boulemane, et au Sud par les régions de Meknès-Tafilalet et de Rabat-SaléZemmour-Zaër (El hajam, 2003). Elle s'étend sur environ 801800 ha, soit près de $1,1 \%$ de la superficie du Maroc. des plantes à la sécurité alimentaire et aux soins de santé primaire n'est plus à démontrer car près de $80 \%$ de la population des pays en développement les utilisent pour se soigner ou pour se nourrir (Allabi et al., 2011) (Nkwanyana et al.,2010). Dans le domaine de la pharmacologie, les connaissances traditionnelles à base de plantes est devenu un outil reconnu dans la recherche de nouvelles sources de médicaments et de produits pharmaceutiques. (Ghosh., 2003) (Sharma et al., 2003). Pour cette raison, aujourd'hui plus que jamais, il est important de sauver le savoir traditionnel populaire de guérison autant que l'utilisation de plantes médicinales et d'offrir des alternatives thérapeutiques communes à traiter les pathologies. L'analyse de la bibliographie médicinale marocaine montre que les données relatives aux plantes médicinales régionales sont très fragmentaires et dispersées. La nécessité de les étudier d'une manière approfondie s'impose d'emblée. Ainsi, nous croyons que le patrimoine de la flore médicinale nécessite un suivi et une évaluation régulière sur le plan qualitatifs et quantitatifs. C'est dans cette optique que nous avons mené une étude ethnobotanique dans la région du Gharb afin de recenser les plantes médicinales et les pratiques médicales dans l'espoir de doter la communauté scientifique d'une base de données sur les plantes médicinales de cette région. Cette étude s'articule autour de deux points : Établir un catalogue de ces plantes et leurs usages thérapeutiques pratiqués par la population locale dans la dite région ; Faire une étude statistiques des informations recueillis concernant ces plantes médicinales et leurs usages. La présente étude, tend en effet a contribué á la connaissance des plantes médicinales de la région du Gharb.

Sa population est estimée à 1656723 habitants dont $56 \%$ en milieu rural, et se répartie sur les trois provinces de la région (Kenitra, Sidi Kacem et Sidi Slimane). Cellesci comprennent 10 communes urbaines et 51 communes rurales (ORMVAG). Le climat de la région est un climat méditerranéen caractérisé par une pluviosité concentrée dans les mois froids de l'année (d'automne au printemps) avec des étés chauds et secs. En effet, la moyenne des 

la région du Gharb, Maroc

températures maximales varie entre 22 et $23^{\circ} \mathrm{C}$ et celle des minimales entre 14 et $17^{\circ} \mathrm{C}$. L'ouverture de la région sur l'océan atlantique lui vaut des précipitations abondantes qui se situent autour d'une moyenne de 450 $\mathrm{mm}$ par an par station au cours des dernières années. La pluviométrie au niveau du Gharb est relativement élevée par rapport aux autres plaines agricoles du Maroc. Elle est concentrée entre le 15 Octobre et le 15 Avril à raison de $90 \%$. Les conditions climatiques de la zone d'étude ont une grande influence sur l'évolution du couvert végétal et des sols et, par conséquent, leur fertilité. La région abrite également deux nappes phréatiques importantes: La maamora d'une superficie d'environ $4000 \mathrm{~km} 2$, et la nappe du Gharb d'une superficie de $390 \mathrm{~km}^{2}$. Le réseau hydrographique de la région est composé d'importants cours d'eau, à savoir l'oued Sebou et ses principaux affluents dont le Beht sur la rive droite. Ces cours d'eau génèrent des apports annuels de l'ordre de $6.10 \mathrm{~m}^{3}$ soit $27 \%$ du potentiel hydrique national mobilisable (Haida, 2000). Du point de vue géologique ; la plaine du Gharb correspond à un fossé d'effondrement, situé entre deux grand ensemble structuraux, qui sont le domaine mésétien primaire et le domaine de la chaîne rifaine au Nord et à l'Est. Cette plaine est marquée principalement par une subsidence continue depuis le Miocène (Michard, 1976). La couverture de la surface de la plaine du Gharb, proprement dit, est formée dans sa totalité par les dépôts du Gharbien dont les terrains

\section{METHODOLOGIE DE TRAVAIL}

Dans le but de dresser un inventaire floristique des espèces médicinales et d'essayer de sauvegarder le maximum d'informations concernant leurs utilisations traditionnelles acquises par la population locale, en prospectant le maximum de zones dans la région étudiée. Nous avons eu recours, d'une part à un plan d'échantillonnage stratifié et d'autre part à une fiche questionnaire comportant des questions précises sur l'informateur (âge, sexe, pratique thérapeutique,...) et les plantes médicinales utilisées par celui-ci (nom vernaculaires, type de maladie traitée, partie utilisée, mode de préparation). A l'aide de 520 fiches questionnaires les enquêtes ethnobotaniques sur le terrain, auprès des informateurs des plantes médicinales, ont été menées pendant deux campagnes 2013 et 2014. Afin de déterminer les différents milieux d'enquêtes nous avons utilisé la méthode d'échantillonnage stratifié (Godron, 1982). Cette technique a pour but d'avoir un anciens forment les zones intermédiaires et les zones basses. Le Gharbien récent constitue les zones de levées alluviales des bords des principaux cours d'eau (Sebou et Beht) (Allouza, 2002). La plaine du Gharb est une zone homogène de terres lourdes résultant des alluvionnements de l'oued Sebou et de ses affluents. En fonction de la texture et des conditions de drainage naturel on distingue trois principaux types de sols, le Dehs, le Tirs, le Dehs lourds et les Sols des anciennes Merjas (Zidane, 2003). Sur le plan économique, la région dispose de potentialités diversifiées qui peuvent être mobilisées pour un développement durable. La région dispose de richesses forestières importantes s'étalant sur une superficie de 125000 ha dont $28,9 \%$ de forêts naturelles. A ce niveau, on dénombre des réserves naturelles et biologiques importantes comme le lac de Sidi Boughaba, la Maâmora et la Merja Zerka. Au niveau agricole, la région jouit d'une gamme variée de sols à haute qualité, de ressources abondantes en eau et occupe une position géographique stratégique caractérisée par sa proximité de l'Europe et des grands centres de consommation (Rabat, Casablanca...). Elle constitue également un réservoir de main-d'œuvre qualifiée, et bénéficie d'un encadrement technique des agricultures de haut niveau, et d'une importante infrastructure routière et ferroviaire permettant d'assurer en toute quiétude le transport des produits agricoles.

inventaire floristique le plus complet possible et de réaliser des enquêtes ethnobotaniques variées d'une zone à une autre dans la région étudiée. Ainsi nous avons repérée 26 stations dont 7 communes urbaines et 19 communes rurales (Figure.1), L'effectif de personnes questionnées dans chaque strate est le même (20 personnes), ce qui totalise 520 personnes. Par cette méthode, nous pensons avoir visité un grand nombre de strates afin d'avoir un maximum d'espèces médicinales utilisées dans la région. Au laboratoire de Biodiversité et Ressources Naturelles de la Faculté des sciences de Kénitra, et à l'aide des la Flore du Maroc (Tome I, II, III). Nous avons déterminé les espèces récoltées sur le terrain afin de dresser la liste complète des espèces médicinales recensées dans la région étudiée. Ensuite, les données recueillies ont été inscrites dans une base de données puis traitées et analysées statistiquement à l'aide du logiciel Microsoft Office « Excel ». 


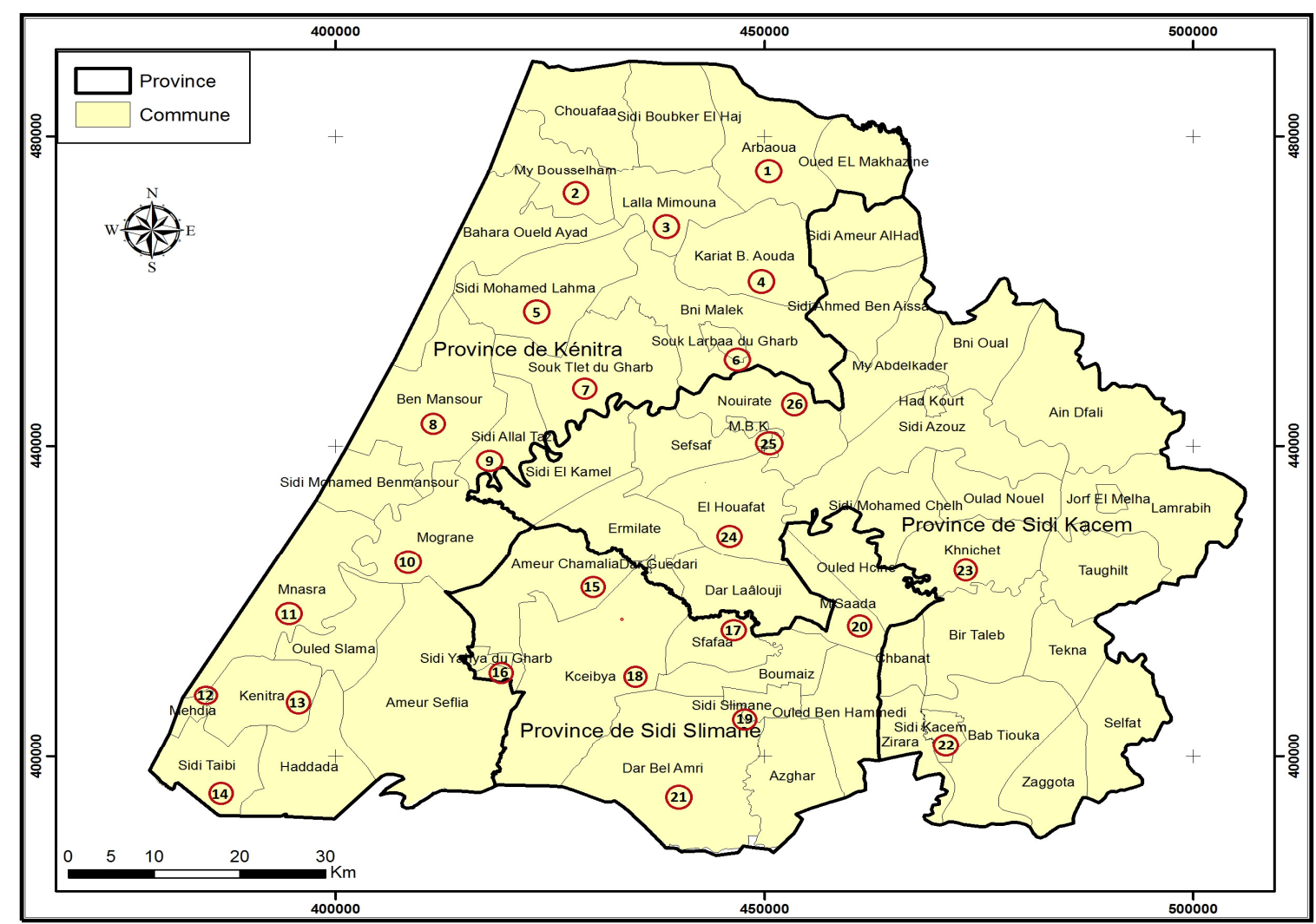

Figure 1 : Carte de répartition des points d'enquêtes ethnobotaniques dans la région de Gharb

\section{RESULTATS ET DISCUSSION}

Utilisation des plantes médicinales selon l'âge : Le traitement des données nous a permis d'établir le tableau 1, qui montre que l'utilisation des plantes médicinales dans la région du Gharb est répandue chez toutes les tranches d'âge, avec une légère dominance chez les personnes âgées de 30 à 39 ans $(29 \%)$. Viennent ensuite les tranches d'âge plus de 50 ans , [40-50] ans, [18-29] ans et enfin celle de moins de 18 ans avec respectivement $26 \%, 24 \%, 19 \%$ et $2 \%$. L'analyse de ces données montre que les personnes de 30 ans et plus ont une fréquence d'utilisation des plantes médicinales de 79 $\%$ contre une fréquence de $21 \%$ chez les personnes d'âge inférieur à 30 ans. La connaissance, des usages des plantes médicinales et leurs propriétés est généralement acquise suite à une longue expérience accumulée et transmise d'une génération à l'autre. La transmission de cette connaissance est en danger actuellement parce qu'elle n'est pas toujours assurée (Anyinam 1995). En effet on remarque que l'expérience accumulée avec l'âge constitue la principale source d'information à l'échelle locale au sujet de l'usage des plantes en médecine traditionnelle. On note aussi une perte d'informations sur les usages thérapeutiques traditionnels, ce qui s'explique par la méfiance de certaines personnes, particulièrement les jeunes, qui ont tendance à ne plus trop croire en cette médecine traditionnelle. Le sexe : La lecture des données du tableau 1 montre que parmi les individus enquêtés $54,6 \%$ sont des femmes et $45,6 \%$ sont des hommes ce qui explique que dans cette région les femmes et les hommes ont recourt à la médecine traditionnelle avec une légère prédominance des femmes. Ceci peut être justifié par le fait que la femme s'occupe de la préparation des recettes pour ses soins et ceux des enfants. Ces résultats sont en cohérence avec les résultats obtenus dans d'autres travaux ethnobotaniques réalisés dans cette région et à l'échelle nationale, Benkhnigue (2010) dans la région de Mechraâ Bel Ksiri et par Mehdioui et Kahouadji (2007) dans la forêt d'Amsittène (Province d'Essaouira) qui ont montré que les femmes sont plus détentrices du savoir phytothérapique traditionnel. 


\section{Bouayyadi et al. J. Appl. Biosci. 2015 Étude floristique et ethnobotanique de la flore médicinale dans}

la région du Gharb, Maroc

Tableau 1 : Profil général des utilisateurs $(\mathrm{N}=520)$

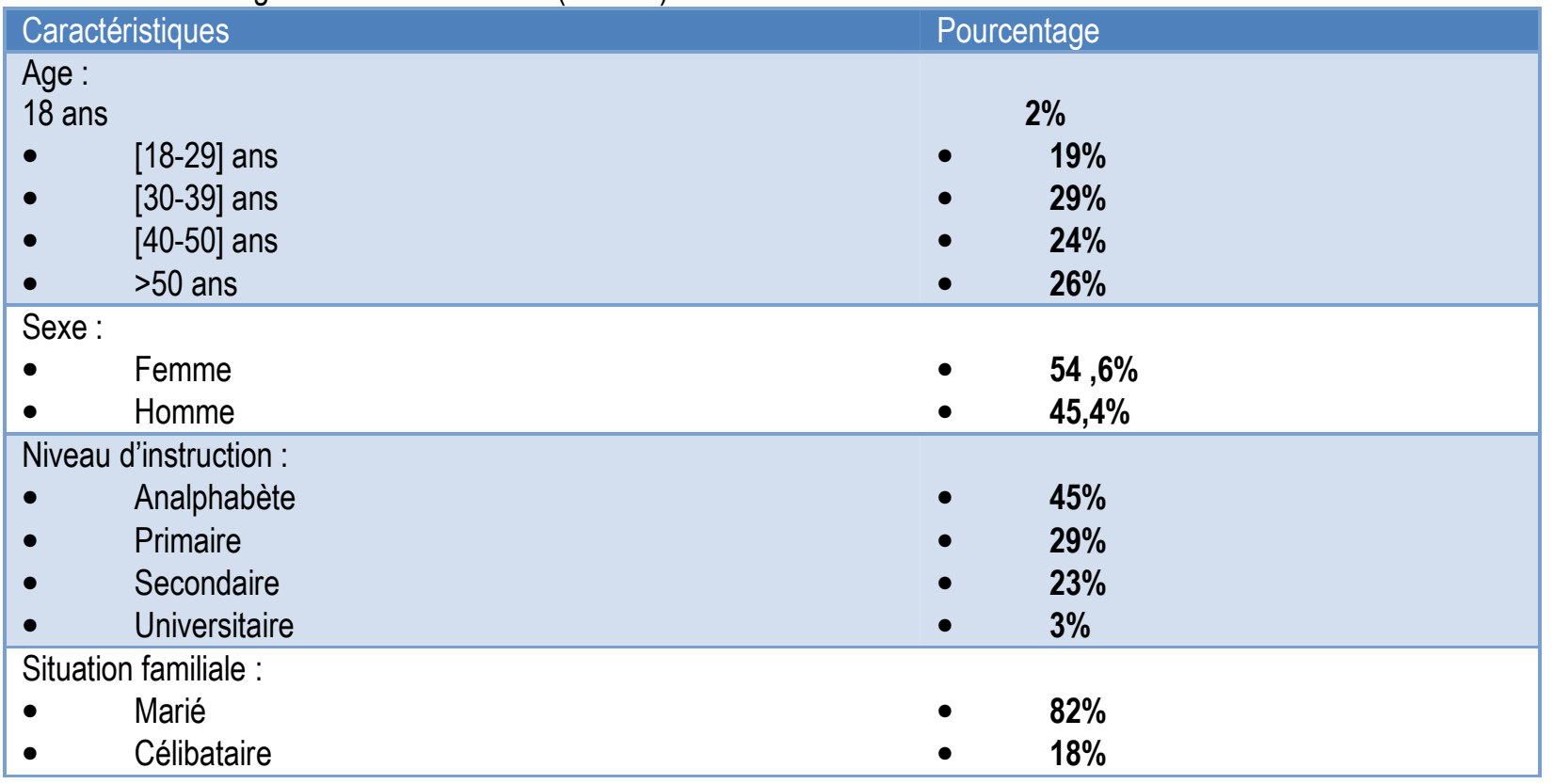

Le niveau d'instruction : L'utilisation des plantes médicinales varie selon le niveau d'instruction. Les analphabètes sont nettement représentés, avec un pourcentage de $45 \%$, que les individus instruits en ce qui concerne l'utilisation des plantes médicinales. Néanmoins les individus ayant un niveau d'études primaire et secondaire ont un pourcentage d'utilisation non négligeable ( $29 \%$ primaire, $23 \%$ secondaire), alors que ceux ayant un niveau d'études universitaire utilisent moins les plantes médicinales (3\%). Ceci s'explique par le fait que la vigilance quant aux effets secondaires potentiels qui peuvent être engendrés par la phytothérapie, augmente avec le niveau d'instruction des individus.

La situation familiale : La plus part des utilisateurs des plantes médicinales sont des personnes mariées avec un pourcentage de $82 \%$ contre $18 \%$ pour les personnes célibataires. Ceci s'explique d'une part par le fait que les parents sont responsables de la santé des membres de la famille, ce sont eux qui donnent les premiers soins en particulier pour leurs enfants, d'autre part ils ont recours à la médecine traditionnelle pour minimiser le coût élevé que ce soit du médicament ou de la consultation du médecin.

Choix entre la phytothérapie et la médecine moderne : L'analyse de la figure 1 montre que la grande majorité de la population utilisent les plantes médicinales comme remède contre les maladies avec un pourcentage cumulatif de $87 \%$ puisque $42 \%$ utilisent la phytothérapie seule et $45 \%$ utilisent la phytothérapie et la médecine moderne contre $13 \%$ de la population qui ne fait confiance qu'à la médecine moderne. Ce recourt à la médecine traditionnelle dans la région du Gharb peut être expliqué d'une part par des raisons culturelles du fait que les gens avaient acquis cette pratique thérapeutique de leurs ancêtres. Ce résultat, corrobore, celui de (Cunningham, 1993) qui rapporte que $80 \%$ de la population Africaine dépend principalement de la médecine traditionnelle pour le traitement des maux. et par des raison socioéconomiques lié le plus souvent à l'enclavement des zones rurales, l'inexistence ou l'état rudimentaire des infrastructures sanitaires, le coût élevé des préparations pharmaceutiques, ainsi que la modicité des revenus des populations (Guedje et al., 2010). 


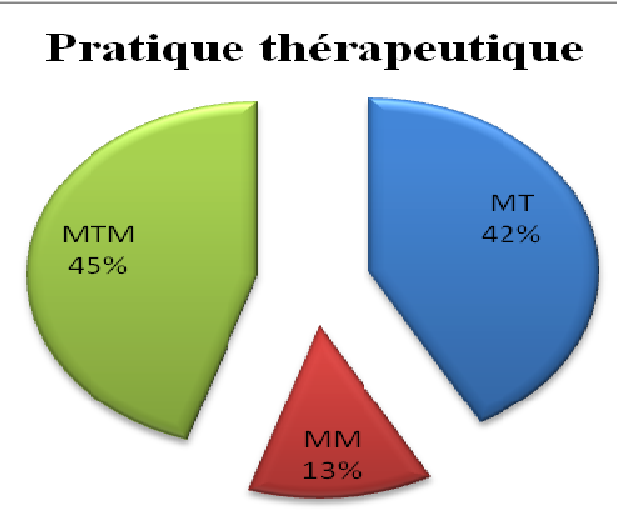

Figure 2: Répartition de la fréquence d’utilisation des plantes médicinales par la pratique thérapeutique dans la région du Gharb

Le mode de préparation : La population de la région du Gharb utilise plusieurs modes de préparations afin d'administrer les principes actif que contient les plantes médicinales. La décoction est le mode d'administration le plus utilisé avec un pourcentage de $35 \%$ (Figure. 2), suivie par la préparation en poudre avec un pourcentage de $26 \%$ et l'utilisation de la plante cru avec un pourcentage de $18,33 \%$, les autres modes de préparation à savoir l'infusion, le cataplasme, la cuisson, la Macération, l'Instillation et autres représentent 20,67\%. Le pourcentage élevé de la préparation en décoction montre que la population locale croit à ce type de préparation et le trouve adéquat pour réchauffer le corps et désinfecter la plante pour annuler l'effet toxique de certaines recettes, mais la décoction peut détruire certains principes actifs des espèces utilisées.

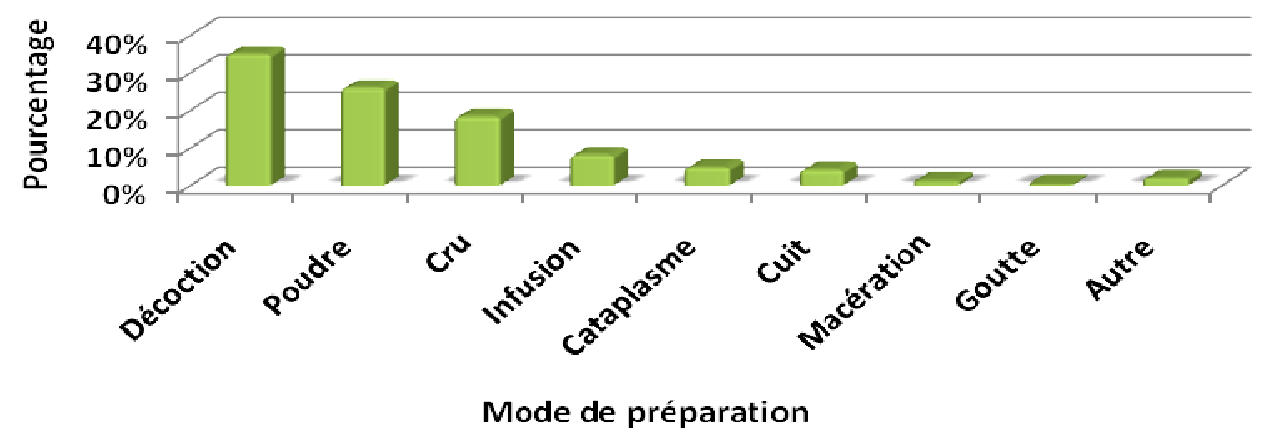

Figure. 3: Répartition de la fréquence d'utilisation des plantes médicinales selon le mode de préparation dans la région du Gharb

La partie utilisée : L'examen de la figure 3 montre que les feuilles sont majoritairement utilisées avec un pourcentage de $38,55 \%$; ensuite viennent les graines $12,20 \%$, les fruits $10,70 \%$, les tiges feuillées $8 \%$, et les Rhizomes 7,25\%. Les parties utilisées restantes (Tige, Plante entière, Écorce, Bulbe, Fleurs, Partie aérienne, Racines et Stigmates de fleurs) sont représentées par un pourcentage cumulatif de $23,6 \%$. Cette fréquence d'utilisation élevé des feuilles, graines et fruit $(61,45 \%)$ peut être expliquée par l'aisance et la rapidité de la récolte (Bistindou, 1986) mais aussi, selon les propos des usagers, par le fait que ces organes soient exposés au soleil ce qui leur procure vertus et bienfaits. L'explication scientifique est le phénomène de photosynthèse qui favorise la biosynthèse et le stockage des métabolites. 


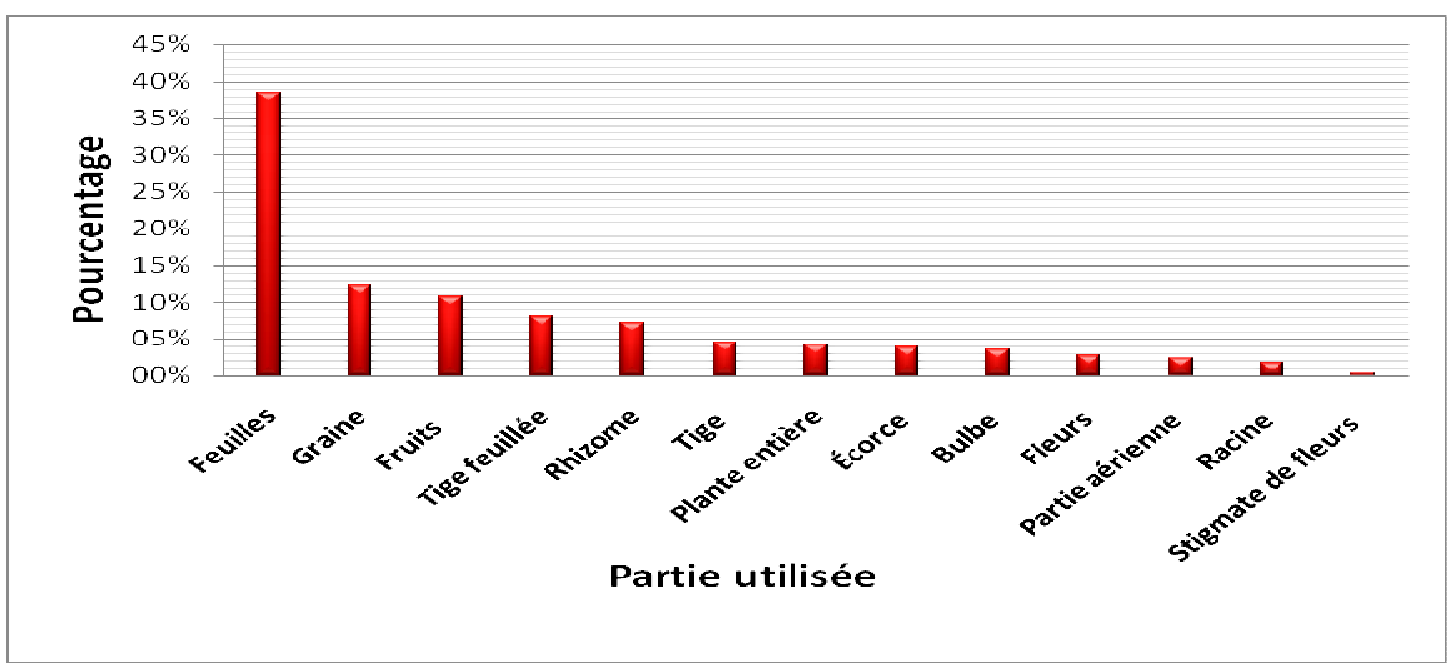

Figure 4 : Répartition de la fréquence d'utilisation des plantes médicinales selon la partie utilisée dans la région du Gharb

Maladies traitées par les plantes médicinales: L'analyse de la figure 4 montre que la population locale de la région du Gharb utilise les plantes médicinales pour traiter principalement les affections du tube digestif avec un pourcentage de $21,84 \%$, métaboliques $(19,67 \%)$, dermatologiques $(18,41 \%)$ et génito-urinaires $(12,27 \%)$, suivies par les affections, respiratoires avec $7,03 \%$, neurologiques $(5,77 \%)$, de l'oreille $(4,87 \%)$ et osteoarticulaires $(4,51 \%)$ le reste englobe un taux de $5,58 \%$. .

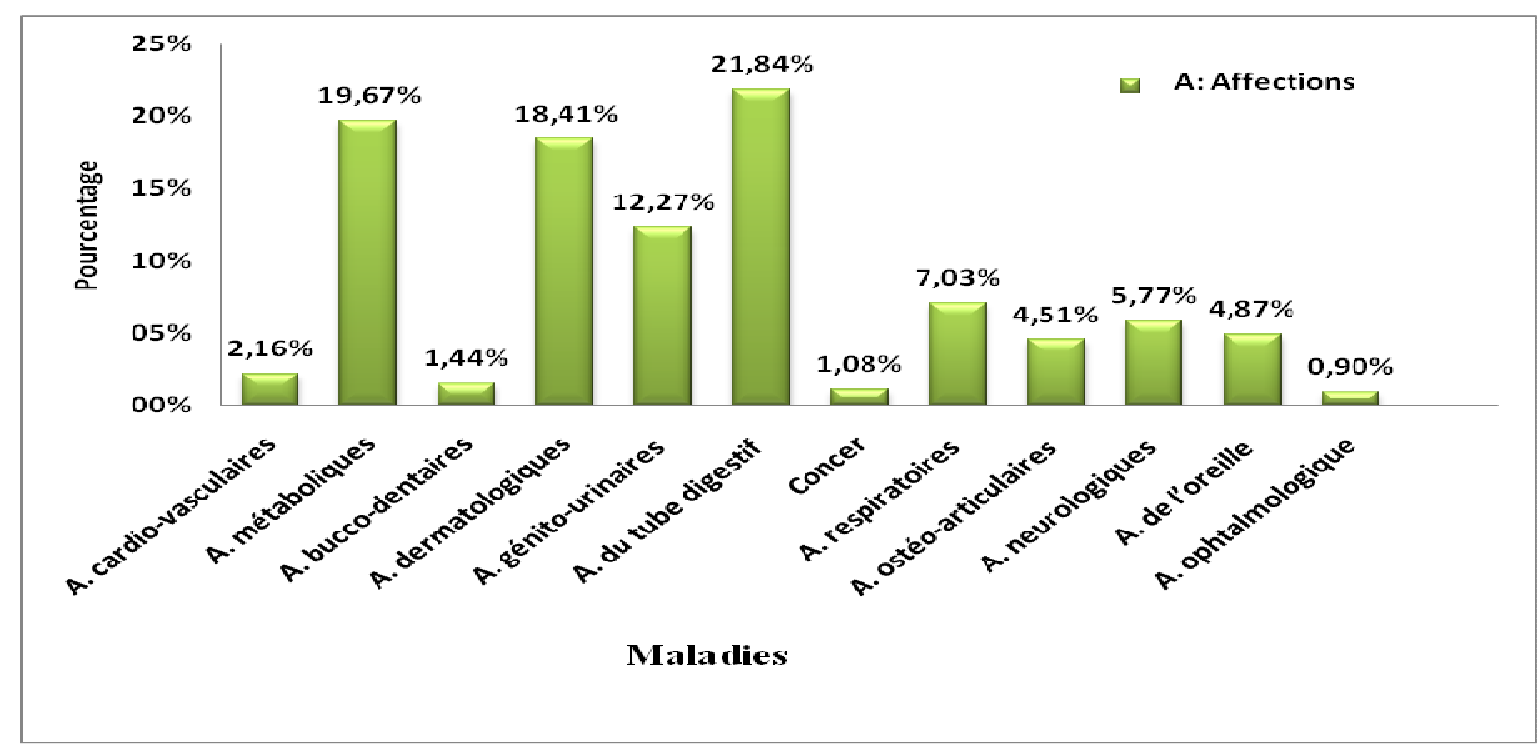

Figure 5: Répartition de la fréquence d'utilisation des plantes médicinales selon les affections traitées dans la région de Gharb

Le mode d'administration : L'administration orale, qui regroupe la majorité des modes de préparation : infusion, macération, décoction, tisane, poudre, cru, cuit, est la plus préconisée (Figure. 5). Ce sont des règles qui répondent au mode de traitement des pathologies les plus rencontrées. Sauf exception faite pour le traitement de certaines affections dermiques et rhumatismales, l'administration orale reste la plus connue et la plus préconisée avec un pourcentage élevé de $63,62 \%$ suivie par le Badigeonnage avec un pourcentage de $18,92 \%$, la fumigation avec $3,95 \%$, le rinçage $(3,34 \%)$ et le massage $(3,12 \%)$ les autres mode d'administration $(7,05 \%)$ sont peu utilisés par la population locale. 


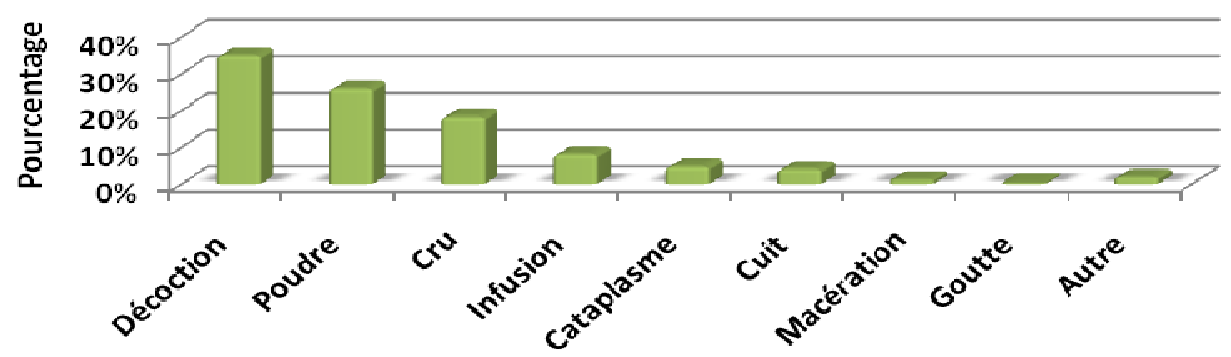

Mode de préparation

Figure 6 : Répartition de la fréquence d'utilisation des plantes médicinales selon la mode d'administration dans la région du Gharb

Analyse floristique : L'analyse floristique des espèces répertoriées montre que 137 espèces médicinales sont utilisées. Elles sont reparties en 116 genres et 57 familles botaniques. De même, le nombre de plantes médicinales marocaines ne dépasse pas 600 espèces (Rejdali, 1996; Bammi \& Douira, 2002), soit $24 \%$ de la flore totale marocaine, ce qui reflète de façon très nette la grande richesse en plantes médicinales de la région étudiée. En outre, les familles botaniques qui dominent la flore médicinale de la région du Gharbe sont les Lamiaceae $(12,4 \%), \quad$ Apiaceae $(8,02 \%), \quad$ Asteraceae $(7,29 \%)$, Fabaceae $(7,29 \%)$, Poaceae $(4,37 \%)$, Rosaceae $(4,37 \%)$, Solanaceae $(2,91 \%)$,Curcubitaceae $(2,91 \%)$. Les autres familles restantes ne comptent qu'une ou deux espèces (70 espèces soit $50,36 \%)$. Le résultat est conforme à celui obtenu par Benkhnigue et Salhi et al (2010) qui ont mené des études sur de villes de la région du Gharb.

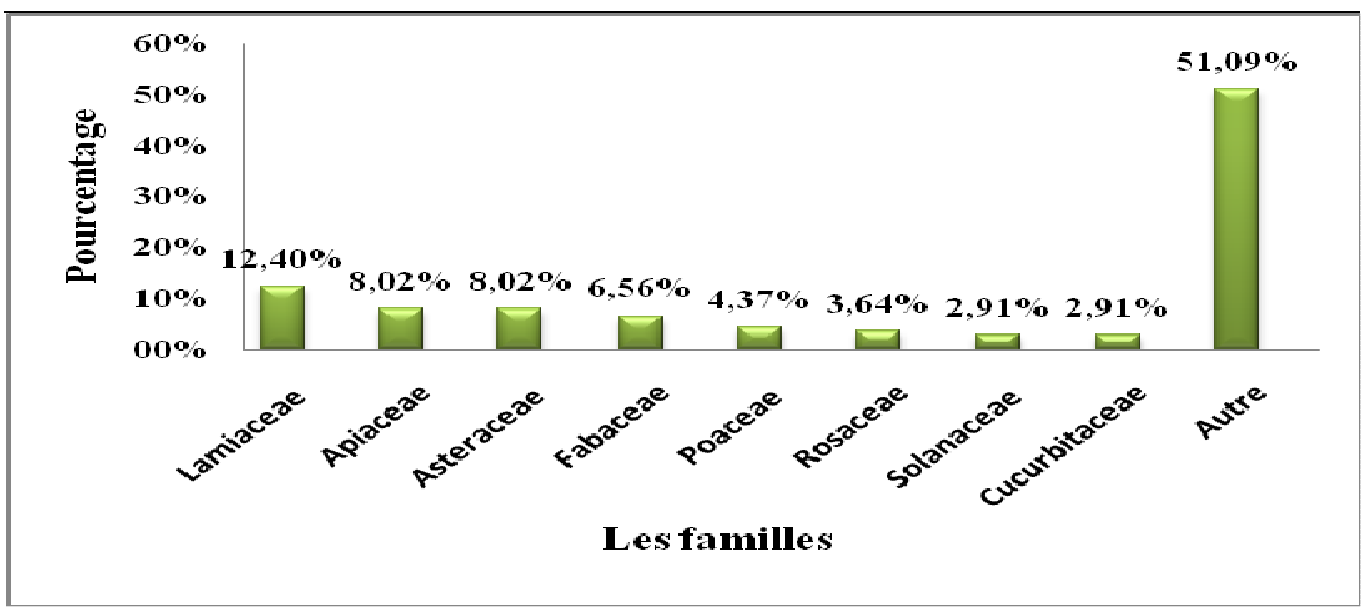

Figure 7: Répartition des familles par le pourcentage des espèces dans la région du Gharb

Plantes médicinales les plus utilisées : Les résultats présentés dans la figure, montre que sur les 137 espèces rencontrées dans la région étudiée, dix sont relativement plus utilisées que les autres espèces en phytothérapie traditionnelle par la population locale. 

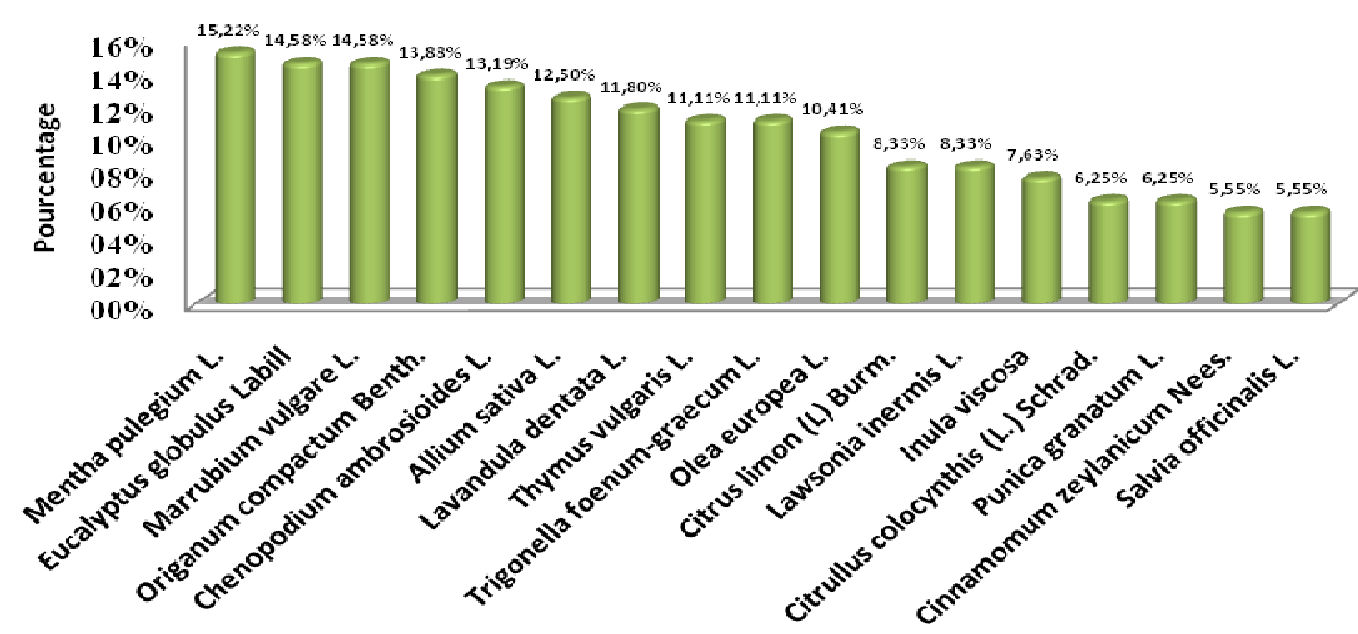

Les espéces

Figure. 8 : Répartition de la fréquence d'utilisation des plantes médicinales les plus utilisées dans la région du Gharb

\section{CONCLUSION}

Au terme de cette étude, il semble que la phytothérapie est incontestablement la médecine la plus adoptée par la population locale de la région du Gharb pour soulager leurs maux quotidiens. II semble aussi qu'il existe encore une implication dans la conservation du savoir-faire populaire en phytothérapie traditionnelle. En effet ce savoir-faire oral a été rapporté dans un catalogue des usages thérapeutiques des plantes médicinales utilisées dans cette région. En outre, l'analyse des résultats obtenus montre que les feuilles constituent la partie la plus utilisée avec un pourcentage de $27 \%$. La décoction (36\%) est le mode de préparation le plus sollicité dans la plupart des recettes, et $77 \%$ de ces recettes sont administrées par voie orale. La plus part des usagers des plantes médicinales sont des personnes mariées avec un pourcentage de $82 \%$ contre $18 \%$ des personnes célibataires. Ces plantes médicinales sont utilisées

\section{RÉFÉRENCES}

Allabi A.C., Busiac K., Ekanmiana V. \& Bakiono F., 2011, The use of medicinal plants in self-care in the Agonlin region of Benin. Journal of Ethnopharmacology, 133, 234-243.

ALLOUZA M., (2002). Evolution morphologique et sédiment logique de la frange littorale de la région de Kenitra: Bilan sédimentaires. Mémoire pour l'obtention du diplôme des études supérieures approfondies, Université Ibn Tofaïl Kenitra, $109 \mathrm{p}$. principalement pour traiter les affections du tube digestif avec un pourcentage de $21,84 \%$. Ces résultats montrent également que les plantes utilisées par la population locale en médecine traditionnelle sont en nombre de 137 espèces reparties en 116 genres et 57 familles botaniques. Dont huit dominent la flore médicinale de cette région notamment: Les Lamiaceae $(12,4 \%)$, Apiaceae $(8,02 \%), \quad$ Asteraceae $(7,29 \%), \quad$ Fabaceae $(7,29 \%)$, Poaceae $(4,37 \%)$, Rosaceae $(4,37 \%)$, Solanaceae $(2,91 \%)$, Curcubitaceae $(2,91 \%)$. Dans la perspective de ce travail nous pensons d'une part contribuer à l'élaboration de nouveaux médicaments naturels en dotant la communauté scientifique d'une base de données sur les plantes médicinales de la région du Gharb et d'autre part sensibiliser les décideurs quant à la sauvegarde de la biodiversité végétale.

Anyinam C., (1995) - Ecology and ethnomedicine. Exploring links between current environmental crisis and indigenous medical practices. Social Science and Médicine, 4, 321-329.

APDN., 2012: Agence pour la Promotion et le Développement du Nord. Étude sur le secteur des Plantes Aromatique et Médicinales dans la Réserve de Biosphère Intercontinentale de la méditerranée. pp 1-130. 
Bammi J et Douira A., (2002)-Les plantes médicinales dans la forêt de l'achach (plateau central, Maroc)-Acta Botanica Malacitana 27: 131-145.

Bellakhdar, J., (1997) - La pharmacopée marocaine traditionnelle. Médecine arabe ancienne et savoirs populaires. Editions Le Fennec, Casablancal Ibis Press. 764 p. Paris.

Benkhnigue O., Zidane L., Fadli M., Elyacoubi H., Rochdi A., et Douira A., (2010-2011)- Étude ethnobotaniques des plantes médicinales dans la région de Mechraa Bel Ksiri (Région du Gharb du Maroc).Acta Bot. Barc. 53 : 191-216.

Bitsindou M., 1986. Enquêtes sur la phytothérapie traditionnelle à Kindamba et Odzala (Congo) et analyse de convergences d'usage des plantes médicinales en Afrique centrale. Thèse de Doctorat. Univ. Libre de Bruxelles. 482 pp.

Cunningham, A.B. 1993. African medicinal plants: Setting priorities at the interface between conservation and primary healthcare. People and Plants Working Paper. UNESCO. Paris. 1-50 pp.

Daget $P$ et Godron M., 1982. Analyse fréquentielle de l'écologie des espèces dans les communautés, édition: Masson. 163 pp.

De Wet H., Nkwanyana M.N. \& van Vuuren S.F., 2010, Medicinal plants used for the treatment of diarrhoea in northern Maputaland, KwaZulu Natal Province, South Africa. Journal of Ethnopharmacology, 130, 2, 284-289.

E.A. Radford, G. Catullo et B. de Montmollin., 2011 Zones importantes pour les plantes en Méditerranée méridionale et orientale. Union internationale pour la conservation de la nature et de ses ressources. Publié par UICN, Gland, Suisse et Málaga, Espagne. pp: 1-124.

El HAJAM S., (2003)," La Planification, outil du Développement Local" "Cas de la Région de Gharb Chrarda Bni Hssen»-ISCAE(Maroc) ; pp : 33-43.

Fennane, M., Ibn Tattou, M., Mathez, J., Ouyahya, A. et El Oualidi, J., (1999) - Flore pratique du Maroc, Vol. I. Trav. Inst. Sci. Rabat, série botanique $36.558 p$.

HAIDA S., (2000) Transport de matière et bilan de l'érosion mécanique et de l'altération chimique dans un basin versant de zone semi-aride : le Sebou. Impacts des variations climatiques et des activités humains Thèse Doct. D'État. Univ. Ibn Tofaïl. Kénitra, 267 p.
Ghosh A (2003) Herbal folk remedies of Bankura and Medinipur districts, West Bengal (India), Indian. J. Trad. Knowledge 2: 393-396.

Guedje N.M., Ntungwen Fokunang C., Tafokou Jiofack R.B., et Fogou Dongmo R., (2010) -Opportunités d'une exploitation soutenue des plantes médicinales dans l'aménagement forestier. Int. J. Biol. Chem. Sci. 4(4) : 1346-1372, August.

Lahsissene H., Kahouadji A., Tijane M., et Hseini S., (2009)-Catalogue des plantes médicinales utilisées dans la région de zaër (Maroc occidental).Lejeunia, BE ISSN 0457-4184.

Mehdioui, R. \& kahouadji, A. 2007. Étude ethnobotanique auprès de la population riveraine de la forêt d'Amsittène: cas de la Commune d'Imi n'Tlit (Province d'Essaouira). Bulletin de l'Institut Scientifique, Rabat, section Sciences de la Vie $n^{\circ} 29: 11-20$.

MICHARD A., (1976), Elément de géologie marocaine. Rabat, Not. Et Mém. Serv. Géol. Maroc, $n^{\circ} 252$, 422P.

Rejdali, M., 1996- La flore du Maroc : état actuel et perspectives de conservation. Diversité biologique et valorisation des plantes médicinales. Actes. éd. 17-22.

Salhi S., Fadli M., Zidane L. et Douira A., 2010. Études floristique et ethnobotanique des plantes médicinales de la ville de Kénitra (Maroc). Lazaroa, 31: 133-146.

Scherrer A.M., Motti R. et Weckerle C. S., 2005. Traditional plant use in the areas of Monte Vesole and Ascea, Cilento National Park (Campania, Southern Italy) J. Ethnopharmacol. 97: 129-143.

Sharma PP, Mujumdar AM. 2003. Traditional knowledge on plants from Toranmal Plateu of Maharashtra. Indian Journal of Traditional Knowledge. 2: 292296.

Sijelmassi A., 1993 - Les plantes médicinales du Maroc, 6ème édition. Fennec. Casablanca. 285 p.

Tabuti J.R.S., Lye K.A. et Dhillion S.S. 2003.Traditional herbal drugs of Bulamogi, Uganda: plants, use and administration. J. Ethnopharmacol. 88: 1944.

ZIDANE, H., (2003), "Étude floristico-écologique des adventices des céréales dans le Gharb, le Saîss, la Tadla et la Haouz (Maroc Occidental) et étude de la compétition entre le blé et l'avoine stérile" université des sciences Ibn Tofaïl Kenitra ; 172 pp. 
CATALOGUE DES PLANTES MEDICINALES DANS LA REGION DU GHARB

\section{Amaranthaceae}

\section{Chenopodium ambrosioides L (Anserine, Mkhinza)}

Le décocté des feuilles est employé, contre les affections gastro-intestinales, la fièvre, le rhum et la dysenterie chez l'enfant comme chez l'adulte. Il est aussi utilisé en cas de douleurs des règles difficiles et le diabète. Le broyat des feuilles d'ansérine (Chenopodium ambrosioides), de khella (Ammi visnaga) et d'aunée officinale (Inula helenium) est utilisée comme badigeonnage contre les démangeaisons et l'gonflement causé en cas d'allergie. Les feuilles d'Ansérine en infusion dans l'eau ou le jus d'orange sont utilisées comme antipyrétique en cas de fièvre ou de douleurs intestinales chez l'enfant. Le décocté de la poudre est utilisé par voie orale contre les affections gastro-intestinales, la typhoïde, et la fièvre.

\section{Amaryllidaceae}

\section{Allium cepa L. (Oignon, Bassala)}

Le broyat du bulbe de l'oignon se met sur les cheveux pour les faire pousser. Le jus de l'ognon cru est utilisé par voie orale contre la toux et pour régler la tension artérielle. En cataplasme les feuilles du bulbe chauffées sont utilisées contre les furoncles. La patte préparée à partir du broyage du bulbe de l'oignon mélangé avec les feuilles de l'Eucalyptus (Eucalyptus globulus Labill), du Marrube blanc (Marrubium vulgare L.), de la Menthe pouliot (Mentha pulegium L.), et de l'Ansérine (Chenopodium ambrosioides L) est indiqué en cataplasme contre les maux de tête. Le jus du bulbe est utilisé en gouttes auriculaires contre les douleurs des oreilles.

\section{Allium sativa L. (Ail, Touma)}

L'ail est consommé cru toute seule ou avec l'huile d'olive contre la toux, l'angine, la pharyngite et le cancer. Le broyat mélangé avec le miel est indiqué en cas de douleurs rénales.

L'extrait du broyat de l'ail avec l'huile d'olive est instillé dans l'oriel contre l'otite et dans le nez en cas de rhinite allergique. Un massage local par l'ail cuite dans l'huile d'olive est conseillé contre les douleurs du dos. L'ail est utilisé comme antifongique par frottement directe sur les parties de la peau touchées par la teigne. L'ail cuite dans l'huile d'olive est indiquée en badigeonnage des cheveux comme antipelliculaire. Le frottement de l'ail sur les yeux est indiqué en cas de démangeaison oculaire.

\section{Apiaceae}

Ammi visnaga Lam. (Khella, Bachnikha)
La fumigation par décoction d'ombelles est utilisées en cas de prurit (démangeaisons de la peau), d'œdème faciale (lorsque le visage gonfle), et d'inflammation ou infection des yeux (Adwa en dialecte). La fumigation par combustion d'ombelles desséchées ou de sa poudre est indiquée contre les douleurs des yeux, le prurit et l'infection des yeux.

Ammodaucus leucotrichus Coss. \&Dur. (Cumin velu, Kmmun es-sofi)

Le décocté des fleurs et des graines est utilisé contre les douleurs abdominales (ou maux de ventre) chez l'enfant et l'adulte. L'infusé des fruits est indiqué contre les douleurs intestinales chez le nourrisson.

Apium graveolens L. (Ache, Krafés)

L'extrait du broyat de feuilles mélangé avec l'eau est indiqué par voie orale en cas de lithiase rénale. Le décocté des feuilles fraiches associées aux feuilles fraiches du persil (Petroselinum sativum Hoffman) est utilisé par voie orale contre les douleurs rénales.

Carum carvi L (Carvi, Karwiya)

La poudre des graines est mâchée cru contre les atteintes de l'estomac.

Conium maculatum L. (Grande ciguë, Ziyyata)

Les feuilles cuites sont utilisées comme complément alimentaire contre le diabète et les maladies du cœur.

\section{Coriandrum sativum L. (Coriandre, Kasbour)}

Une cuillère à café de la poudre des graines dans un verre d'eau est conseillée en cas de diarrhée. Le décocté des tiges feuillées est conseillé pour la désintoxication et la purification du sang.

\section{Cuminum cyminum L. (Cumin, Kamoun)}

Le décocté des grains est indiqué par voie orale lors des règles douloureuses. Une cuillère à café de la poudre des graines est consommée cru contre la diarrhée. La poudre mélangée avec le miel et Poudre de Coquelicot (Papaver rhoeas L) séché "Aker Fassi" est conseillée par voie orale pour nettoyer le foie et l'organisme.

\section{Daucus carota L. (Carotte, Khizou)}

Le décocté de la poudre des graines est utilisé par voie orale contre le cancer. Le jus de carotte associer à la poudre du Henné (Lawsonia inermis L, du Trigonella foenum-graecum) et de l'alun (Chebba en dialecte) est utilisé en badigeonnage contre l'eczéma.

Foeniculum vulgare L. (Fenouil, Nafaâ, Bessbass) Le décocté des graines est indiqué avec le lait contre la constipation et seul pour soulager les crises de l'asthme. Le décocté des feuilles desséchées est utilisé par voie orale en cas de refroidissement.

Petroselinum sativum Hoffman (Persil, Maâdnous) 
L'infusé de la poudre des graines est utilisée par voie orale en cas de lithiase rénale (cailloux ou pierres dans les reins). Le décocté de la tige feuillée ou du rhizome est indiqué par voie orale, contre la lithiase rénale et le refroidissement, et comme aphrodisiaque. II est aussi bon pour nettoyer les reins et la détoxification du foie.

Pimpinella anisum L. (Anis vert, Habat hlawa)

La tisane préparée à partir de la poudre de la plante entière desséchée est indiquée par voie orale en cas de ballonnement au ventre (ballonnement abdominal) par les gaz.

\section{Apocynaceae}

\section{Caralluma europaea (Caralluma, Darmouss)}

La poudre ou les feuilles, associées avec le leben, est préconisée par voie orle dans le traitement des kystes génitales.

Nerium oleander L. (Laurier rose, ddefla)

Le rinçage des pieds par le décocté des feuilles est indiqué contre le diabète et le rhumatisme articulaire. Le frottement direct et local de la gencive par les feuilles du Laurier rose est indiqué en cas de gingivite (inflammation de la gencive). Le frottement de la peau par les feuilles du Laurier rose est utilisé contre Le lichen plan (maladie inflammatoire touchant la peau). Une fumigation par combustion des feuilles est efficace contre le gonflement.

\section{Arecaceae}

\section{Chamaerops humilis L. (Palmier, Palmier nain,} Doum, Jummakh)

Le décocté du rhizome est conseillé après les repas en cas de l'ulcère gastrique.

\section{Aristolochiaceae}

Aristolochia longa L. (Aristoloche, Berez'tem)

Le décocté du rhizome de l'Aristoloche est utilisé une fois par jours par voie orale en cas d'atteinte par le cancer. La poudre du rhizome est utilisée comme antiseptique et cicatrisant des plaies et des blessures.

\section{Asparagaceae}

Asparagus officinalis L. (Asperge, Seckoum)

Le décocté du rhizome est indiqué par voie orale contre les douleurs intestinales et le cancer.

L'infusé est utilisé par voie orale contre le rhumatisme.

Drimia maritima L. (Scille, L'aânssela)

Le patient atteint de la fièvre typhoïde passe toute la nuit couché sur le broyat de la plante entière placé sur le matelas.

Asteraceae

Artemisia arborescens L (Absinthe arborescente, Chiba)
Le décocté de la tige feuillée et du lait est utilisé par voie orale contre le refroidissement, et il est utilisé à jeune le matin pendant trois jours pour laver l'estomac en cas d'ingestion de substance toxique. L'infusé préparé à partir de la tige feuillée et l'huile d'olive est instillé dans les oriels contre l'otite.

Artemisia herba alba Asso (Armoise blanche, Chih) Le décocté de la tige feuillée permet d'éradiquer les parasites intestinaux ou vers et il est utilisé dans le traitement du cancer. L'infusé des graines d'armoise est utilisée comme un vermifuge pour se débarrasser des parasites intestinaux.

Atractylis gummifera L. (Chardon à glu, Addad)

Une solution préparée par mélange de l'eau et la poudre des racines associée aux feuilles du Henné (Lawsonia inermis L.) est préconisée par rinçage du corps contre le prurit. Le masque naturel préparé à partir de la poudre des racines et de l'eau est conseillé par badigeonnage contre la chute des cheveux.

\section{Centaurea marocana Bail. (Centauré, Tafgha)}

Le fruit est utilisé cru ou cuit par voie orale contre la gastrite (inflammation de la paroi ou muqueuse de l'estomac).

\section{Cynara cardunculus L. (Cardon, Khorchef)}

Le décocté du fruit mélangé avec le miel est indiqué en cas d'infection du foie par un virus. Après une fumigation par la vapeur de la décoction des racines du chardon associées avec les feuilles de l'Eucalyptus globulus Labill (Kalito, kalitous, Eucalyptus), les feuilles de Glycyrrhiza Foetida (Réglisse foetide, Guenfdo), les graines desséchées de Silybum marianum L. (Chardon Marie, Tawra) et les feuilles du Marrubium vulgare L.( merriwta) le décocté est utilisé par voie orale contre la fièvre typhoïde (Mkelfa en dialecte).

\section{Cynara scolymus L. (Artichaut cultivé, L-qôq)}

Le fruit est consommé crut en cas d'affection de l'estomac.

Inula viscosa $L$. (Aunée visqueuse, Bagraman) Le décocté des feuilles est utilisé par voie orale contre les affections intestinales et génitale ; et par massage contre l'eczéma Le décocté de la plante entière mélangé avec le sel et l'alun (Chebba en dialecte) est indiqué par gargarisme pour calmer les douleurs dentaires. Le broyat chauffé est indiqué en cataplasme en cas de douleurs rhumatismales. La poudre des feuilles desséchées est indiquée contre les blessures, les furoncles et les abcès cancéreux.

Matricaria chamomilla L. (Camomille, Babounj)

Le décocté des feuilles et des fleurs est indiquées chez l'adulte et l'enfant contre la jaunisse (ictère cutanéomuqueux). Trois gouttes du vinaigre de la 
camomille dans un verre d'eau est indiquée par voie orale chez les personnes âgées et les adultes contre l'allergie et l'asthme.

Ormenis mixta (L.) Dumt (Camomille du Gharb, Hellâla)

Le décocté de la partie aérienne de la camomille du Gharb est utilisé en rinçage contre les douleurs buccales. La décoction des capitules est employée par voie orale, contre les douleurs de l'estomac et comme laxatif. Les fleurs de la camomille du Gharb sont utilisées par fumigation contre les affections génitales. Le broyat des feuilles est employé en cataplasme pour traiter les hémorroïdes.

\section{Scolymus hispanicus L. (Scolyme d'Espagne, Garnina)}

L'infusé du rhizome dans l'eau chaude est indiqué par voie orale contre la gastrite. Les feuilles sont consommés cru ou avec l'alimentation en cas de refroidissement.

\section{Silybum marianum L. (Chardon Marie, Tawra)}

Le décocté des graines desséchées est indiqué contre les douleurs rénales.

\section{Brassicaceae}

Brassica rapa L. (Navet, Left)

Le broyat des feuilles fraiches est utilisé en cataplasme contre les maux de tête et la fièvre.

Lepidium sativum L. (Cressonnette, Hobb rchad)

Les graines desséchées sont indiqués cru avec le lait par voie orale contre le refroidissement et pendant La période du post-partum (après l'accouchement).

\section{Sinapis alba (Moutarde blanche, Khardal)}

La poudre des graines mélangée avec l'eau est utilisée par rinçage pour calmer les douleurs rhumatismales.

\section{Cactaceae}

Opuntia ficus-indica L. (Cactus, Zaâboul)

La poudre de raquette de figuier de Barbarie est indiquée par voie orale contre le diabète. Les jeunes raquettes sont également utilisées en association avec le Ghassoul (une argile saponifère, qui a un pouvoir nettoyant) par badigeonnage pour lisser et adoucir les cheveux. Le fruit est consommé cru comme complément alimentaire chez les sujets atteins de l'anémie. Le broyat des jeunes tiges est utilisé par badigeonnage pour renforcer les cheveux. Capparaceae Capparis spinosa L. (Câprier, Kabbar)

La poudre du fruit desséché mélangée avec le miel est indiquée contre le refroidissement, les problèmes de la respiration et comme aphrodisiaque. La poudre est conseillée avec le thé chez les femmes souffrant de refroidissement et des douleurs des règles.

Caryophyllaceae

Herniaria glabra L. (Sabline rouge, Harras lahjar)
Le décocté de la plante entière est indiqué en cas de lithiase rénale.

Chenopodiaceae

Spinacia oleracea L. (Epinard, Selq)

Les feuilles fraiches sont bouillies avec la Mauve (Malva sylvestris L.) et utilisé par voie orale contre le refroidissement.

\section{Cucurbitaceae}

Citrullus colocynthis (L.) Schrad. (Cologhinte, Lhdej) Les graines séchées sont employées en cas d'hyperglycémie (deux graines par jour avec un verre de petit lait acidifié le matin). Le fruit coupé en deux est frotté contre la peau pour traiter l'eczéma et toute sorte de mycose. Pour apaiser les douleurs rhumatismales on met le talon du pied dans le fruit tranché en deux pendant 20 min. Le fruit vidé de ses graines et du liquide d'accompagnement est remplis du lait et on le laisse toute la nuit se refroidir à l'air libre et le matin on boit ce lait pour traiter les douleurs intestinales et le refroidissement. Des suppositoires préparés à partir du fruit et le coton imbibé d'huile d'olive sont utilisés par voix rectale pour traiter toute sorte de refroidissement. L'extrait des fleurs se verse en une goutte dans les yeux pour les nettoyer. Cucumus sativus L. (Concombre, Khiyar)

Des tranches du fruit frais sont déposées directement sur les yeux en cas de cernes.

Ecballium elaterium (L.) A. Rich. (Concombre d'âne, Faggous el-hemar)

Le jus du fruit frais est instillé directement dans le nez contre la jaunisse.

Lagenaria siceraria (Molina) Standl (Courgette de salé, El garâa-slâwiya)

L'écorce du fruit test desséché et utilisé en cataplasme pour apaiser les douleurs de la migraine.

Cupressaceae

Cuperssus Sempervirens (Cyprès, Zembale)

Un bain de bouche par le décocté des feuilles fraiches est indiqué contre les douleurs dentaires.

Tetraclinis articulata(Vahl.)Masters (Thuya, Arâr)

Le broyat des feuilles est appliqué sur la peau en cataplasme contre l'eczéma.

Cyperaceae

Cyperus rotondus $\mathrm{L}$. (Souchet rond, Tamusayt)

L'inhalation de la vapeur de Tamusayt est employée en cas d'atteinte de migraine.

Euphorbiaceae

Euphorbia falacta L. (Euphorbe en faux, Hayyat ennufus)

La poudre de la plante entière desséchée est utilisée avec l'alimentation contre le refroidissement et comme préventif des maladies. 
Mercurialis annua L (Mercuriale annuelle, Harrayga malsa)

Le décocté de mercuriale est utilisé contre le refroidissent, la stérilité, et les atteintes de l'utérus. La broyat de Mercuriale fraiche mélangé avec de l'eau est utilisé en badigeonnage pour faire pousser les cheveux. Ricinus communis L. (Ricin, Krank)

Les feuilles se mettent en cataplasme directe sur la tête contre la fièvre et les maux de tête

La fumigation par la vapeur (l'aarga) des feuilles du Ricin, de la carotte (Daucus carota), de l'oranger (Citrus sinensis L.), d'Eucalyptus (Eucalyptus globulus Labill), et du Marrube blanc (Marrubium vulgare L.) en décoction est indiquée en cas d'atteinte de typhoïde.

\section{Fabaceae}

\section{Acacia raddiana Savi (Acacia saharien, Talh)}

La poudre des feuilles desséchées est utilisée par application locale en cataplasme en cas de brulure cutané.

\section{Cassia senna L. (Séné d'Alexandrie, Sannâ haram)}

Le décocté des feuilles associées avec les fleurs de Rosa damascena Mill. Est indiqué par voie orale en cas de douleurs de l'appareil urinaire. La poudre des feuilles desséchées est indiquée le matin avec le miel et la nuit avec l'eau pendant trois jours en cas d'intoxication par ingestion de substance toxique (Toukal en dialecte dans la culture marocaine). L'infusé de trois feuilles facilite le transite intestinal en cas de constipation.

\section{Cicer arietinum L. (Pois chiche, Hommess)}

L'infusé des graines dans l'eau est utilisé par voie orale contre la jaunisse. La poudre des graines est indiquée en cas de lithiase rénale.

Glycyrrhiza Foetida (Réglisse foetide, Guenfdo)

Le décocté des feuilles est utilisé par voie orale contre la jaunisse et la migraine. Le patient atteint de la jaunisse passe toute la nuit couché sur les feuilles fraiches de Glycyrrhiza Foetida placées sur le matelas. Les feuilles fraiches sont aussi utilisées par cataplasme sur la tête contre la jaunisse et la migraine.

Glycyrrhiza glabra L. (Réglisse, Arq Sûs)

La tisane préparée par infusion des racines de la Réglisse est indiquée par voie orale contre l'ulcère de l'estomac, l'asthme et le rhumatisme.

Medicago sativa L. (Luzerne, Fassa)

Les feuilles fraiches sont bouillies et consommés comme fertilisant.

Retama monosperma Boiss. (Retam, Rrtam)

Le décocté de la tige est utilisé pour traiter les maladies de la gencive.

Trigonella foenum-graecum L. (Fenugrec, Halba)
La poudre mélangée avec le miel est utilisée par voie orale pour traiter les maladies de l'estomac et le ballonnement des intestins et pour augmenter le lait chez la femme, par badigeonnage pour traiter le lentigo (Leklef en dialecte). La poudre mélangée avec le petit lait acide est employée pour traiter le diabète.

Vicia faba L. (Fève, El-fûl)

La poudre des graines séchées est consommée le matin seule ou bien suivie du jaune d'un œuf ou d'un verre de thé contre la tuberculose pulmonaire. La poudre mélangée avec l'Ail (Allium sativa L.) et l'huile d'olive est conseillé le matin contre la tuberculose et le refroidissement.

\section{Fagaceae}

Quercus suber L. (Chéne-liége, Dbagh)

Le décocté de l'écorce des racines est indiqué par voie orale contre l'ulcère de l'estomac.

\section{Gentianaceae}

Centaurium ambellatum (L.) (Petite centaurée, Kosset el haya)

La poudre des feuilles associée à la poudre du fenugrec (Trigonella foenum-graecum L) (Halba en dialecte) et mélangée au miel est indiquée par voie orale contre les douleurs de l'estomac.

\section{Geraniaceae}

\section{Pelargunium Capitatum (Gernium rosat, Laatercha)}

La tisane préparée par décoction des feuilles fraiches est utilisée par voie orale contre les douleurs intestinales et le stress.

\section{Iridaceae}

\section{Crocus sativus (Safran vrai, Za'afran horr)}

La poudre des stigmates desséchés est indiquée en application locale contre les blessures.

Juglandaceae

Juglans regia L. (Noyer, Sswâk)

L'infusé préparé à partir du Noyer frais est indiqué en cas de douleurs gastriques. Le noyer desséché est utilisé par moxibustion (Alkaye en dialecte) pour traiter les verrues. Lamiaceae

Ajuga iva (L.) Schreber (Ivette, Bugle, Chendgûra) Le décocté est utilisé par voie orale contre la migraine et en cas de morsure de chien. Et par rinçage en cas de démangeaisons de la peau (hakka). L'infusé de d'Ajuga iva (L.) (Chendgûra en dialecte) est utilisé contre le refroidissement. La poudre d'Ajuga iva (L.) desséché mélangée avec du miel et la poudre de Trigonella foenum-graecum L (Halba), Centaurium spicatum (L.) (Kesset Ihayya) et Marrubium vulgare L. (merriwta en dialecte) en cas d'intoxication du système digestif (toukkal dans la culture marocaine).

Calamintha officinalis L. (Calament, Manta) 
La décoction du calament est utilisée par voie orale contre la grippe et la fièvre.

Lavandula dentata L. (Lavande à feuille denté, Lakhzama)

Le décocté des feuilles desséchés est utilisé par voie orale contre les douleurs abdominales, le refroidissement, les douleurs intestinales, les douleurs des règles, les difficultés respiratoires et comme régulateur du cycle des règles. La poudre des feuilles desséchées mélangée avec l'huile d'olive ou du miel est utilisée contre l'incontinence urinaire chez les enfants énurésie, la diarrhée et l'allergie. Des suppositoires préparées à partir de la poudre des feuilles et l'huile d'olives sont indiquées en cas d'affection de l'utérus. Les graines desséchées et broyées avec le jus d'orange sont indiquées contre la grippe et le refroidissement.

Lavandula stoechas L. (Lavande stoechade, Halhal) Le décocté est utilisé par voie orale contre le diabète et le refroidissement.

La fumigation par la vapeur ( L'aarga en dialecte) des feuilles de la Lavande stoechade associées avec les feuilles de l'Eucalyptus (Eucalyptus globulus Labill), du Ricin (Ricinus communis L.), du Citronnier (Citrus limon (L) Burm), du Bourrache langue de beauf (Isan tawr) (Borago officinalis), et de la ronce des bois (Rubus fiuticosus.) est indiquée la fièvre et la typhoïde.

Marrubium vulgare L. (Marrube blanc, Marriwta elharra)

Le décocté est utilisé par voie orale contre la toux, la bronchite, La tuberculose pulmonaire et l'asthme. Après décoction la plante est utilisée par cataplasme directe sur le lieu du male en cas de douleurs abdominales ou du dos, et elle se met sur la tête comme remède à l'allergie qui se manifeste par l'écoulement des yeux et du nez. La poudre préparée à partir du Marrube blanc, du Girofle (Eugenia caryophyllata Thunb) et le Mandragore (Mandragora autumnalis Bertolt) mélangée avec du miel est employée pour traiter la stérilité chez les femmes. Les feuilles du Marrube blanc et raisin sec (Zbib en dialecte) broyés avec de l'eau sont indiqués après filtration contre le cancer. Le broyat du Marrube blanc se met dans le nez et sur la tête de l'enfant en cas de perte d'appétit. Avec les feuilles du Marrube blanc mâchés on brosse les dents et la gencive en cas de gingivite.

Mentha pulegium L. (Menthe pouliot, Fliyou)

La tige feuillée en décoction dans l'eau, le lait ou le thé est utilisée contre le Rhume, la toux, la bronchite, les douleurs de la vessie, les douleurs abdominales et le refroidissement. La vapeur de la tige feuillée en décoction est utilisée en cas d'affection de l'utérus chez la femme et aussi contre le rhume. La poudre des feuilles desséchées est utilisée comme cataplasme après un massage de la poitrine avec l'huile d'olive contre la toux. La patte préparée à partir de la poudre des feuilles et l'huiles d'olives est employée en massage contre les douleurs rhumatismales.

Mentha officinalis L. (Menthe à feuilles rondes,
Marseta) Une tisane préparée à partir de l'infusion de trois feuilles dans un verre d'eaux est indiquée pour l'amincissement. Les feuilles fraiches sont utilisées en cataplasme locale contre les furoncles.

Mentha viridis $L$. (Menthe verte, Na'an, Liqamâ)

Le frottement local des feuilles est efficace contre les verrues (toulal en dialecte).

Mentha x piperita L. (Menthe poivrée, Na'na el-âabdi) Le liquide obtenu par broyage des feuilles est instillé dans les yeux en cas d'allergie oculaire.

Ocimum basilicum L. (Basilic, Lahbaq)

La tisane préparée par décoction des feuilles du Basilic est conseillée comme laxatif contre la constipation.

Origanum compactum Benth (Origan, Zaâter)

La tige feuillée en décoction, est utilisée contre les affections gastro-intestinales, la grippe, le rhume, les règles douloureuses et comme hypoglycémiant. L'infusé est utilisé contre les douleurs abdominales et comme fortifiant de la mémoire et aide à la concentration. L'hinalation de la vapeur du Zaâter associé à Fliyou (Mentha pulegium L.) et Lakhzama (Lavandula dentata L.) est conseillée en cas d'atteinte de migraine. La poudre mélangée avec l'hile d'olive se met directement sur l'anus pour traiter les hémorroïdes. Le décocté de la poudre de l'origan dans le lait est utilisé contre la grippe et le rhume. La poudre est utilisée comme antiseptique et cicatrisant des plaies et des blessures. La pâte préparée à partir de la poudre du Zaâter, la poudre de Lakhzama (Lavandula dentata L.) et la poudre de Nigella sativa L (Haba sawda) mélangé avec l'huile d'olive est employée en badigeonnage contre la chute des cheveux.

Origanum majorana L. (Marjolaine, Merdedûch)

Le décocté des feuilles est utilisé par voie orale en cas de douleurs menstruelles, de froid dans l'utérus, de maux de l'estomac et contre la toux.

Rosmarinus officinalis L (Romarin, Iklil al-jabal, Azir) Les feuilles, en infusion ou en décoction, sont employés contre les douleurs d'estomac, des intestins, les règles douloureuses et comme hypoglycémiant. En usage externe, la poudre de la tige desséchée mélangée avec la farine et l'huile d'olive sont appliquées comme antiallergique et contre dans l'appareil génitale femelle. L'infusion de romarin associé avec Chenopodium ambrosioides L (Mkhinza, Anserine) est utilisée en cas 
d'atteintes de la Typhoïde et de la vessie L'infusé des feuilles est indiqué par voie orale contre l'allergie du printemps et comme stimulant de la circulation sanguine. Salvia officinalis L. (Sauge officinale, Salmiya) L'infusé préparé par macération des feuilles dans l'eau est indiquée contre le stress, la nervosité et L'hypotension artérielle, (baisse ou chute de tension). Le décocté des feuilles seul ou avec le thé est indiqué contre la lithiase rénale, le diabète, le refroidissement, et les règles douloureuses. Une tisane préparée par infusion d'une cuillerée à café dans un verre d'eau est préconisée contre l'insolation, les frissons et la stérilité.

Salvia verbenaca (L.) Briq. (Fausse verveine, Khiyata) Les feuilles fraîches broyées sont utilisées, en cataplasme, sur les plaies, les abcès vidés et les brulures pour faciliter leur cicatrisation. Le décocté des feuilles est utilisé par voie orale pour apaiser les douleurs de l'estomac.

\section{Teucrium polium L. (Germandrée polium, J'idya)} La fumigation par la vapeur de la décoction des feuilles desséchées est conseillée contre les douleurs dentaires et l'œdème facial (gonflement du visage).

Thymus vulgaris L. (Thym, Zâaytra)

Le décocté des feuilles fraiches est utilisé en cas d'affection de l'appareil génital de l'homme et pour traiter l'acidité dans l'estomac. Le décocté des feuilles séchées est employé contre les douleurs de l'estomac, la toux et le refroidissement. Le décocté à base du thym et de Rosmarinus officinalis $L$ (Romarin) est utilisé contre la dysenterie (diarrhée). Le décocté à base du thym et d'Origanum compactum Benth (Zaâter) est utilisé par voie orale comme régulateur du taux du cholestérol dans le sang.

\section{Lauraceae \\ Cinnamomum zeylanicum Nees. (Cannelle de Ceylan, Qarfa)}

L'infusé de l'écorce est indiqué par voie orale en cas de règles douloureuses.

\section{Linaceae}

Linum usitatissimum L. (Lin, Zariât lkatan)

Une graine desséchée est administrée par voie orale deux fois par semaine contre le diabète. La poudre des graines desséchés et grillés est utilisée avec de l'eau par voie orale pour baisser le taux du cholestérol dans le corps. La poudre des graines est indiquée par voie orale avec le miel ou le lait contre l'allergie. La poudre des graines associer avec la poudre du Fenouil (Foeniculum vulgare L.), de Nigelle (Nigella sativa L) et de l'Anis vert (Pimpinella anisum L.), mélangée avec le miel est utilisée contre le refroidissement et les douleurs intestinales. Lythraceae

\section{Lawsonia inermis L. (Henné, Henna)}

Une tisane préparée par infusion des feuilles du Henné est employé contre les douleurs de l'estomac, l'ulcère, les maux et l'inflammation des intestins, l'acidité gastrique. La pâte préparée à partir de la poudre des feuilles du henné mélangée avec de l'eau est utilisée en application locale pour la coloration et le traitement des cheveux, elle est aussi réputée par son usage antipelliculaire, antiseptique et cicatrisant des plaies, des blessures et des brulures. Le décocté du henné et de Chenopodium ambrosioides $L$ (Mkhinza, Anserine) mélangé avec du vinaigre est utilisé par voie orale en cas d'atteinte par la migraine. La poudre des feuilles du henné mélangé avec I'huile d'olive est utilisée en badigeonnage contre la chute des cheveux et comme antipelliculaire. En association avec le vinaigre ou (Qetrane en dialecte) la poudre du henné traite certaine dermatose (eczéma).

\section{Punica granatum L. (Grenadier, Er-rummân)}

Le décocté préparé à partir de l'écorce du fruit ou de sa poudre est prescris par voie orale en cas de problèmes gastro-intestinale (estomac et intestins) et de douleurs abdominales. La poudre de l'écorce du fruit est utilisée directement ou en décoction avec du thé par voie orale pour calmer les douleurs de l'estomac, elle est aussi prescrite comme cicatrisant des plaies et des blessures et en cas de brulure. La tisane, préparée par infusion de la poudre de l'écorce, est prescrite par voie buccale pour traiter. Les vers intestinaux, Ténia, et l'ulcère. La poudre de l'écorce desséchée est mélangée avec le Lawsonia inermis L. (Henna, Henné) et Lavandula dentata L. (Lakhzama, Lavande) d'une quantité d'environ une poignée de chacune pour être prescrite en badigeonnage, comme anti-chute des cheveux.

\section{Malvaceae}

Malva sylvestris L. (Mauve, L-khobiza, El baqûla)

Le décocté des feuilles est utilisé contre les affections du tube digestifs et comme anti-allergique. Malva Sylvestris L. cuite est réputée comme un aliment qui a des vertus laxatif, régulateur de la tension artérielle, de nettoyage des intestins et contre le refroidissement.

\section{Moraceae}

Ficus carica L (Figues sèches, El karmôss, Chriha) Le figuier séché cuit avec l'ail et mélangé avec l'huile d'olive est employé par voie orale contre la toux. Le fruit bouilli dans l'eau est utilisé en cataplasme contre les hémorroïdes.

\section{Myrtaceae}

Eucalyptus globulus Labill (Eucalyptus, Kalito, kalitous)

Le décocté des feuilles est utilisé par voix orale contre les douleurs de l'estomac, les affections des voix 
respiratoires, les infections urinaires la toux et les douleurs des règles. Par inhalation et cataplasme les feuilles d'eucalyptus apaisent les maux de tête.

Eugenia caryophyllata Thunb (Girofle, Qoronfel)

Le décocté est utilisé comme bain de bouche pour traiter les doleurs dentaires.

L'infusé est employé par voie orale comme antiallergique, antalgique (calme les maux de tête), aphrodisiaque et comme anesthésiant. La poudre seule ou mélangé avec la poudre du Skenjbîr (Zingiber officinale Roxb.) est utilisée en badigeonnage pour calmer les douleurs rhumatismales du dos.

\section{Myrtus communis L. (Myrte, Rihan)}

Le décocté de la tige feuillée est utilisé par voie orale contre les douleurs intestinales et les hémorroïdes.

\section{Nitrariaceae}

Peganum harmala L. (Harmel, Harmel)

La poudre des graines mélangée avec (Allathukh en dialicte) chaud est indiquée par badigeonnage contre l'eczéma

\section{Oleaceae}

Olea europaea L. (Olivier, Zitoun)

Le décocté des feuilles est utilisé par voix orale contre le diabète, les maux dentaires, les aphtes, l'hypertension et le rhumatisme. L'huile extraite à partir du fruit est utilisée avec l'ail contre les affections respiratoires et la toux, mélangée avec l'ail et la figue sèche traite les crises d'asthme et les infections respiratoire. L'huile d'olive avec le soufre est efficace contre la maladie dermique le prurit (hakka en dialecte). Un massage par l'huile d'olive soulage les douleurs du dos et du cou.

\section{Pinaceae}

Cedrus atlantica (Endl) (Carrière Cèdre de l'Atlas, Qitran)

L'huile essentielle (Qitran en dialecte) est utilisée par voie orale contre le cancer.

\section{Pinus pinaster Aiton (Pin maritime, Tayda)}

La poudre de l'écorce est utilisée directement ou avec du beurre clarifié (le smen en dialecte) en cataplasme en cas de brulure de la peau.

\section{Plantaginaceae}

Plantago major L. (Grand plantain, L-messâssa)

Les feuilles de s'utilisent comme cataplasme contre les abcès.

\section{Poaceae}

Agropyrum repens P. Beauv. (Chiendent, Njem)

La poudre de la plante entière desséchée est utilisée par voie orale avec le thé contre le reflux gastrique et en cas de poumon perforé.

Oryza sativa L. (Riz, Rûz)
Le masque de visage préparé à partir de la poudre des graines desséchées mélangée avec la farine et l'eau est utilisé en badigeonnage comme anti-taches.

Pennisetum typhoides (Burn.) Stapf et Hubb. (Millet, Ïllân)

La poudre des graines desséchées associé avec le miel ou l'infusé des graines dans l'eau sont indiquée par voie orale pour le renforcement des os et en cas de fracture. Phragmites communis Trin. (Roseau, L-qaseb)

L'infusé de la poudre préparée à partir du rhizome est indiqué par rinçage contre le prurit (démangeaison de la peau) du cuir chevelu et de la peau.

Triticum turgidum L. (Blé, Zraa)

Le son (l'enveloppe du caryopse, séparée de l'amande) du blé mélangé avec le jaune de l'œuf est utilisé comme purifiant du visage.

Zea mays L. (Maïs, Chwachi, Zerab kbal, Hrir ed-dra) La farine du fruit entre dans la préparation du pain comme complément alimentaire en cas d'anémie. Le décocté des soies des épis de maïs est indiqué en cas de cirrhose du foie et des douleurs rénales.

\section{Polygonaceae}

Polygonum aviculare L. (Rénouée, Wedmo)

La poudre préparée à partir de la plante entière (partie aérienne et racines) facilite le drainage des furoncles et permet la cicatrisation des plaies. Le décocté est indiqué par voie orale pour le traitement des douleurs du dos.

\section{Rumex acetosa L. (Oscille, Hommida)}

La poudre du rhizome desséché est mélangée avec du beurre clarifié (le smen en dialecte) pour être utilisée en badigeonnage contre le cancer.

Portulacaceae

Portulaca oleracea L. (Pourpier potager, Rejla)

Les feuilles cuites sont considérées comme complément alimentaire pour stimuler et tonifier le cœur.

\section{Ranunculaceae}

\section{Clematis flammula L. (Clématite, Nar el-barda)}

Le broyat de la partie aérienne de la Clématite se met sous forme de cataplasme à l'aide d'un bouchon au niveau de la cheville de l'individu soufrant de la sciatique (Bûzelûm en dialecte), le lendemain en enlevant ce cataplasme un liquide jaune s'écoule du pied du malade ce qui en résulte un soulagement des douleurs.

Nigella sativa L. (Nigelle, Sanouje)

La poudre des graines desséchées mélange avec le miel est indiquée contre les maladies du foie, les douleurs rhumatismales et du dos, de l'estomac et de l'intestin.

\section{Rhamnaceae}

Ziziphus lotus (L.) Lam. (Jujubier, Sedra, Nnbeg)

Le décocté des feuilles fraiches est indiqué contre la diarrhée. Le décocté du rhizome est utilisé par voie orale 
contre a toux et la bronchite. Le décocté du rhizome et le fruit du jujubier associer avec les racines de la Réglisse (Glycyrrhiza glabra L.) est efficace contre la lithiase rénale.

\section{Rosaceae}

Crataegus laciniata Ucr et C. monogyna Jacq (Aubépine, Zu'rûr)

L'infusé de la poudre des feuilles desséchées associé au miel est indiqué par voie orale en cas de problèmes cardiaque et de respiration.

Eriobotrya japonica (Thunb.) Lindl. (Néflier du japon, Lemzah)

Une fumigation des feuilles de Lemzah, de Sferjel (Cydonia oblonga Mill.) et de Marriwta el-harra (Marrubium vulgare L) est efficace contre la fièvre typhoïde.

Prunus amygdalus Stokes var amara DC. (Amandier amer, Louz Ihar)

La poudre du fruit cru est mélangée avec le miel pour traiter la l'allergie.

Rosa damascena Mill. (Rose pale, El-ward)

Les fleurs sont préconisées en cataplasme contre les maux de tête.

Rubus ulmifolius Schott. (Ronce, Aoulek)

Le décocté des feuilles est utilisé par rinçage locale pour raffermir la peau du visage. Le décocté des feuilles en association avec les feuilles de l'eucalyptus, du Cardon (Cynara cardunculus L.), de la lavande (Lavandula dentata L.) et du Marrube blanc (Marrubium vulgare L.) est indiqué par voie orale contre la céphalée.

Rubiaceae

Coffea arbica L. (Café, Qahwa)

La poudre des graines du café est utilisée en cataplasme contre les brulures cutanées légères.

\section{Rubia tinctorium L (Garance, Fouwa)}

Les feuilles cuites sont indiqués comme complément alimentaire en cas d'anémie. La poudre du rhizome mélangé avec le miel est indiquée par voie orale contre l'anémie.

\section{Rutaceae}

Citrus limon (L) Burm (Citronnier, El-hammed)

Le jus de citron est utilisé par voix orale contre les douleurs intestinales, en gargarisme pour les angines, et en mettant des rondelles directement sur le franc il apaise les maux de tête et donne aussi un effet antipyrétique. Le jus de citron mélangé avec le miel et l'huile d'olive ou avec du persille et de l'aile est utilisé pour traiter les néphrolithiases. Le jus de citron est utilisé en gouttes oculaires en cas d'irritation des yeux. Le jus de citron mélangé avec de la farine et du sucre a des bienfaits pour la peau du visage.
Citrus medica L. (Cédratier, Trunj)

Le jus du fruit mélangé avec l'eau de fleur d'oranger pure est indiqué par voie orale en cas de lithiase biliaire (Pierre dans la vésicule biliaire). Un massage local par le jus du fruit est efficace contre les douleurs articulaires et le gonflement des pieds.

\section{Citrus sinensis (L) (Oranger doux, Leymun)}

Le masque préparé par l'association de la poudre de l'écorce du fruit desséchée mélangé avec un yaourt nature non sucré, est utilisé en badigeonnage pour traiter l'acné. Le décocté du jus du fruit mélangé avec un œuf est utilisé par voie orale la nuit contre la grippe.

Schisandraceae

Illicium verum (Anis etoilé, Badiana)

Le décocté est utilise pour traiter les pathologies du système digestif.

\section{Scrophulariaceae}

Verbascum sinuatum L. (Molène, Muslih al-ândar)

La poudre préparée à partir de la partie aérienne de la plante desséchée est utilisée par application locale contre les blessures cutanée.

\section{Solanaceae}

Capsicum annuum L. (Paprika, tahmira)

La poudre préparée à partir du fruit desséché, est indiquée en cataplasme comme hémostatique local pour l'arrêt des saignements de blessures cutanées superficielles.

Capsicum frutescens L. (Piment enragé, Ssûdaniya, Felfel harr)

Le décocté de la poudre du Piment enragé est indiqué en massage en cas d'atteinte de douleurs rhumatismales. La poudre du Piment enragé, mélangée avec le broyat de l'ail, est conseillée en application locale contre la teigne du cuir chevelu.

Mandragora autumnalis Bertolt (Mandragore, Bayed el-ghôl)

A partir de la poudre du rhizome du Mandragore et du coton on prépare des suppositoires utilisés par voie rectale contre les hémorroïdes et par voie vaginale contre les problèmes de l'utérus.

Solanum tuberosum L. (Pomme de terre, Batâta)

Le rhizome bouillie et broyé avec un œuf est utilisé comme masque de soin du visage. Des tranches du rhizome se mettent directement sur la peau en cas de brulure.

\section{Tamaricaceae}

Tamarix aphylla (L.) Karst. (Tamaris, Adba)

La poudre des graines desséchées mélangée avec le miel est utilisée par voie orale contre la gastrite (inflammation de la paroi de l'estomac).

Tamarix arabica (Tamarix, tarfa) 
La poudre de la tige feuillée associée à la poudre des feuilles du Ricin (Ricinus communis L.) et de l'écorce du fruit du Grenadier (Punica granatum L.) est utilisée en cataplasme contre les douleurs de la tête.

\section{Theaceae}

\section{Camellia sinensis Link. (Thé, Atây)}

L'infusé des feuilles du thé est utilisé par voie orale contre les douleurs abdominales (ou maux de ventre). La poudre est indiquée en cataplasme en cas de blessure ou de brulure.

Thymelaeaceae

\section{Thymelaea lythroides (Passerine, Metnan)}

Le décocté est utilisé par voie orale pour faciliter le drainage en cas d'infection de l'appareil urinaire.

\section{Urticaceae}

Urtica dioica L. (Grande ortie, L-hurrîga)

La grande ortie est utilisée directement sur la peau pour calmer les douleurs rhumatismales et en badigeonnage elle est utilisée comme fortifiant et anti-chutte des cheveux

\section{Valerianaceae}

Valeriana celtica L. (Nard celtique, Ssunbul)

La poudre des feuilles desséchées associée à La poudre des graines du Harmel (Peganum harmala L.), des feuilles du Thuya (Tetraclinis articulata), des feuilles de Lakhzama (Lavandula dentata L.), de la tige feuillée du Myrte (Myrtus communis L.), des feuilles du Henna (Lawsonia inermis L.), du Girofle (Eugenia caryophyllata Thunb), et des fleures de la Rose pale (Rosa damascena Mill.) mélangée avec de l'eau tiède est utilisée en cataplasme comme antipelliculaire et en cas de fracture.

\section{Verbenaceae}

Lippia citriodora H. B. \& K. (Verveine odorante, Lwiza) L'infusé des feuilles desséchées est conseillé par voie orale en cas de stresse, de diarrhée et de douleurs intestinales il est aussi considéré comme désinfectant et antispasmodique. Le décocté des feuilles desséchées est indiqué contre la grippe, le rhumatisme, le refroidissement et les douleurs intestinales.

Vitex agnus-castus L. (Gattilier, L-kharwaâ)

L'extrait des feuilles et du fruit est utilisé en badigeonnage comme antichute et pour renforcer les cheveux.

\section{Vitaceae}

Vitis vinifera L. (Vigne, Dalya)

Les feuilles sont utilisées fraiches par application local en cataplasme contre les furoncles.

Xanthorrhoeaceae
Aloe socotrina Lamk. (Aloès, Sebar, Ssabra)

L'extrait visqueux des feuilles est utilisé en badigeonnage après un bain pour adoucir la peau du visage et les cheveux. Le gel obtenu à partir du broyat des feuilles est utilisé pour l'hydratation des cheveux secs.

Asphodelus microcarpus Salzm. \& Viv. (Asphodèle, Blaluz).

L'huile infusée par macération des racines de l'asphodèle dans de l'huile d'olive est instillé dans les oreilles contre l'otite inflammation ou infection des oreilles).

\section{Zingiberaceae}

Alpinia officinarum Hance (Galanga officinale, Khôdenjâl)

Le décocté de la poudre du rhizome desséché est indiqué contre le diabète, le refroidissement, et incontinence urinaire. Le décocté des racines en association avec les racines de Rubus ulmifolius Schott. (Ronce, Aoulek), les racines de Agropyrum repens P. (Chiendent, Njem) et les racines de Juncus maritimus (smar) ; est utilisé par voie orale comme aphrodisiaque.

Curcuma xanthorrhiza Roxb. (Curcuma, Kharqum) La poudre du rhizome desséché mélangé avec le ou l'eau et le laissé macéré toute la nuit est utilisé par voie orale contre la jaunisse.

Zingiber officinale Rosc (Gingembret, Zanjabîl, Skenjbîr)

L'infusé préparé à partir de l'écorce ou de sa poudre et l'eau chaude ou le lait chaud est indiquée par voie orale, contre le diabète, le rhumatisme, les douleurs des règles, la diarrhée, le rhume, problème de la gorge, comme aphrodisiaque, stimulant de la circulation sanguine et pour renforcer l'estomac (renforcement du muscle du sphincter). La patte préparée par la poudre du Gingembre et l'huile d'olive est utilisée par massage local contre les douleurs du dos. La poudre du rhizome est préconisée par voie orale en association avec l'eau, le lait chaud ou le jus d'orange contre la grippe, le rhum, la toux et le refroidissement, et pour l'épuration sanguine. La poudre est aussi utilisée en cataplasme local pour ces effets antalgiques contre les douleurs du dos.

\section{Zygophyllaceae}

Zygophyllum gaetulum Emb. \& Maire (zygophylle, 'aggaya)

La poudre préparée à partie de la plante entière desséchée est utilisée par voie orale avec de l'eau contre les douleurs de l'estomac. Le décocté des tiges desséchées est utilisé par voie orale en cas d'intoxication des intestins et contre les règles douloureuses. 